\title{
RESULTS OF QUESTIONING PATIENTS WITH NON-ALCOHOLIC FATTY LIVER DISEASE IN THE CONDITIONS OF DENTAL PRACTICE
}

DOI: $10.36740 /$ WLek202103122

\author{
Dmitry V. Emelyanov \\ V. N. KARAZIN KHARKIV NATIONAL UNIVERSITY, KHARKIV, UKRAINE
}

\begin{abstract}
The aim: Is to develop and test questionnaires allowing systematization of the main dental complaints and knowledge on hygienic oral care in patients with non-alcoholic fatty liver disease (NAFLD) given their individual characteristics.

Materials and methods: The developed questionnaires and database have been tested on 36 patients with NAFLD and 10 patients of the control group.

Results: As a result of the study, it appears that the main dental complaints in patients with NAFLD are dry mouth and the associated need for frequent mouth rinsing with water, bleeding gums, mainly during teeth brushing, distortion of taste and bad breath. It has been noted that only $33.3 \%$ of patients regularly brush their teeth every morning and evening, while $25.0 \%$ of respondents brush their teeth only once a day. It is also characteristic that more than $25.0 \%$ of patients with NAFLD use mainly carbohydrate foods in their diet, and the smoking rate among this group is at least $58.0 \%$.

Conclusions: The use of this questionnaire makes it necessary to take into account all the "bottlenecks" in selection of measures for dental disease prevention in NAFLD, which currently is of high relevance and necessity.
\end{abstract}

KEY WORDS: non-alcoholic fatty liver disease, periodontitis, dryness questionnaire

Wiad Lek. 2021;74(3 p.l):504-507

\section{INTRODUCTION}

The relevance of studying combined somatic and dental diseases is beyond doubt, since the internal organs pathology in patients can cause development of structural and functional changes in the oral cavity and have a significant impact on their course and prognosis [1-3]. According to various scientific studies, there is a close relationship between changes in the oral cavity and the digestive system pathology, which is due to the anatomical and morphofunctional unity. One of the most globally widespread somatic diseases is non-alcoholic fatty liver disease (NAFLD), which currently takes the form of a pandemia [4-5].

Today NAFLD is considered as a chronic disease with morphological changes in the form of steatosis, non-alcoholic steatohepatitis and fibrosis (cirrhosis) [6-7]. Despite the extensive study of this disease, there is no data on any single etiological factor in NAFLD development, but several risk factors inherent in the metabolic syndrome are distinguished, namely: obesity, hypodynamia, insulin resistance, hypertriglyceridemia, hypertension, age-related aspects [8-9].

There are a number of works containing data that prove the relationship between periodontal disease and NAFLD. Thus, in 2017, W. Alazawi with a group of colleagues studying the relationship between chronic periodontitis, NAFLD and diabetes showed that these conditions had common risk factors [10]. The authors noted that patients with steatosis were significantly more likely to suffer from chronic periodontitis, with more severe stages of NAFLD having a high correlation with the severity of periodontitis.
A similar study was conducted by a group of scientists who studied periodontal disease in the inhabitants of Pomerania (Aderonke A. Akinkugbe, 2017). Researchers noted that the prevalence of periodontal disease was related to the incidence of NAFLD [11].

Inflammatory periodontal diseases are known to have a multifactorial nature and arise when several risk factors are combined, such as poor oral hygiene, smoking, nutritional faults, genetic predisposition, and somatic diseases. However, the aggressive effect of Porphyromonas gingivalis in case of oral hygiene violation is recognized as the main etiological factor, while NAFLD can serve as a background and significantly aggravate the course of dental pathology [12]. The influence of P. gingivalis on NAFLD pathogenesis was noted by Japanese scientists in their study (Masato Yoneda and et., 2012). The results of the study showed that the frequency of $P$. gingivalis occurrence in patients with NAFLD was statistically higher than in somatically healthy patients [13]. It was found that P. gingivalis could cause significant changes in the composition of the entire oral cavity microflora, stimulate the proteinase activity of neutrophils and increase development of periodontal tissue destruction, as well as exert a systemic effect associated with their toxic and immunopathogenetic effects. It is characteristic that $\mathrm{P}$. gingivalis in the same way affects the intestinal microflora, since the intestinal composition of the microbiota reacts very actively and shifts in response to changes in a person's lifestyle (diseases, eating pattern, mood, etc.). 
Table 1. Example of the questionnaire for patients involved into the study

\begin{tabular}{|c|c|c|c|c|}
\hline \multicolumn{5}{|c|}{$\begin{array}{l}\text { Dear patient, please fill out this questionnaire (choose the answer to the question), carry out self-assessment for the presence and severity of dental } \\
\text { complaints, which largely reflect the state of your dental health. This will help us in diagnostics and make the treatment process more effective! }\end{array}$} \\
\hline Seq. No. & Complaints and questions & Never & Often & Always \\
\hline 1 & Do you ever have a dry mouth sensation? & & & \\
\hline 2 & Do you feel dryness in your mouth, regardless of food intake? & & & \\
\hline 3 & Do you wake up at night to drink water due to a dryness sensation? & & & \\
\hline 4 & Do you have a need to rinse your mouth with water throughout the day? & & & \\
\hline 5 & Do you feel taste distortion? & & & \\
\hline 6 & Do you have bad breath? & & & \\
\hline 7 & Do you feel pain and itching in your gums? & & & \\
\hline 8 & $\begin{array}{l}\text { Do you have gum bleeding? } \\
\text { - when brushing teeth } \\
\text { - when eating } \\
\text { - spontaneous bleeding }\end{array}$ & & & \\
\hline 9 & Do you have communication difficulties due to the above complaints? & & & \\
\hline 10 & Are you undergoing periodontal treatment? & & & \\
\hline 11 & Do you visit your dentist regularly for professional oral hygiene? & & & \\
\hline 12 & Do you use dental hygiene products recommended by your dentist? & & & \\
\hline 13 & Do you brush your teeth 2 times a day? & & & \\
\hline 14 & Do you rinse your mouth after eating? & & & \\
\hline 15 & How often do carbohydrate foods prevail in your diet? & & & \\
\hline 16 & How often do you drink sugary carbonated beverages? & & & \\
\hline 18 & Do you often eat foods containing probiotics? & & & \\
\hline 19 & Do you smoke and how often? & & & \\
\hline
\end{tabular}

In this regard, development of questionnaires to identify the prevalence of major dental complaints and perform daily hygienic care of the oral cavity will allow the formation of a program aimed at prevention of possible dental disorders in this category of patients.

\section{THE AIM}

The aim of this work is to develop and test questionnaires allowing systematization of the main dental complaints and knowledge on hygienic oral care in patients with NAFLD given their individual characteristics.

\section{MATERIALS AND METHODS}

Detection of dental complaints, data on the nature of a diet and methods of individual oral hygiene, bad habits has been carried out though interviewing and questioning patients with self-completion (Table I). When compiling the list of questions, the necessary requirements for the questionnaire have been taken into account: simplicity and unambiguity of questions; possibility of self-completion by the patient; brevity; registration of modern domestic and international dental questionnaires.

The questions in the questionnaire are represented in certain blocks, namely:

Block 1. Basic dental complaints (dry mouth, the need for mouth rinsing with water due to dryness, taste distortion, bad breath, pain and discomfort in the gums, bleeding gums, difficulties in communicating with people due to the above complaints);

Block 2. This block includes questions regarding visits to the dentist and individual oral hygiene (regularity of visits to the dentist for professional oral hygiene, selection of means and items of oral hygiene, frequency of teeth brushing);

Block 3. This block concerns nutrition issues (nature of food, drinking sugary carbonated beverages, eating probiotic products, bad habits).

To process complaints and questionnaire data, an electronic database has been developed based on SPSS 21 software, which allows entering and storing data collected during the survey. The questionnaires have been tested on 36 respondents with verified diagnosis of NAFLD and 10 somatically healthy patients (control group). At the time of the survey, none of the patients in the control group had any complaints or anamnesis data of respiratory and cardiovascular system diseases, endocrine diseases, acute inflammatory processes in the oral cavity, and also none of them was taking any medications at the time of the study. All the results of somatically healthy patients collected during the study completely coincided with those that can be found in modern literature, which made it possible to use these data as reference control values. 


\section{RESULTS}

When questioning and testing questionnaires, the following results have been obtained: for example, the most common complaint is dry mouth, which is suffered by $91.6 \%$ of patients, and $64.9 \%$ of them complain of constant dry mouth. Patients note that the dryness sensation appears gradually and increases over time. A large half of the interviewed patients with this complaint notes that dryness cannot be eliminated, since all means turn out to be ineffective. Almost $42.0 \%$ of respondents note that night dryness causes sleep interruption, there is a need for water drinking and mouth rinsing with water, even though everyone agrees on low effectiveness of such procedures, since they bring immediate relief, but its duration is no longer than several hours. Dryness causes discomfort when chewing and swallowing food. The respondents note that the dryness sensation is accompanied by a change in the saliva properties, which becomes viscous, foamy, and unable to moisten the oral cavity.

Besides, $83.33 \%$ of patients with NAFLD complain of bad breath (halitosis), and 21 patients (58.33\%) continuously have this complaint, while only 6 patients say that they never have such sensations. Halitosis cause inconvenience in communication with others, bring discomfort, reduce the quality of patients' lives. Most patients associate the halitosis phenomenon with a feeling of lack of saliva and its inability to excrete food debris.

Most patients with NAFLD also suffer from taste distortion when eating (25 patients), pain and itching in the gums (25 patients), and $88.8 \%$ of the respondents note bleeding gums, mainly when brushing teeth. It should be noted that none of the patients surveyed notes spontaneous bleeding of the gums.

When processing data in the questionnaires of somatically healthy patients, it has been noted that only 2 patients (20\%) complain of periodic dry mouth and inconveniences associated with it, and 5 respondents suffer from halitosis, 1 of them claims constant halitosis. Also, a frequent complaint among somatically healthy patients is bleeding gums, $40 \%$ - when brushing teeth, and 1 patient - when eating solid foods (hard vegetables, fruits).

It has been found that $77.7 \%$ of patients with NAFLD have been undergoing periodontal treatment over the past 5 years, but only $27.7 \%$ of all regularly visit the dentist for professional oral hygiene and follow the recommendations, which is probably due to low motivation regarding own dental health. Besides, it should be noted that only 33.3\% regularly brush their teeth 2 times a day (every morning and evening), while most patients brush their teeth once a day or once every two days (5 respondents).

When asking more specific questions about the nature of food, it has been revealed that carbohydrate foods predominate in the diet of most patients with NAFLD, and half of the respondents drink sugary carbonated beverages, but only $19.4 \%$ of surveyed report their regular consumption.

We also received disturbing data regarding cigarette smoking. Thus, in the group of patients with NAFLD, more than $80.0 \%$ of patients smoke, and $58.33 \%$ of the respondents have a very high degree of nicotine addiction.

\section{DISCUSSION}

Our research confirms once again that all pathology of internal organs is directly displayed in the oral cavity $[14,15]$. The developed questionnaire allows evaluating the main complaints of patients, identifying deficiencies in hygienic oral care, and assessing the nature of a diet to achieve effective control of dental status.

It was noted that the most frequent complaints for the patients with NAFLD were xerostomia, halitosis and distortion of taste sensitivity, which is probably associated with a violation of the qualitative and quantitative parameters of the oral fluid. It is known that the oral fluid performs many functions, the main of which are protective (moisturizing the tissues of the oral cavity, utilizing food and epithelial debris, forming a barrier of antibodies and other active substances), trophic (maintaining constant hydration and physiological regeneration of the mucosa), etc. Since NAFLD is often accompanied by overweight, symptoms of carbohydrate metabolism disorders and arterial hypertension, xerostomia can be caused not only by the action of pathological processes in the body, but also by taking medications prescribed for this. [16]. As a result, during xerostomia, the oral mucosa is affected, burning sensations appear, taste changes and bad breath appears, and the risk of periodontal disease increases [17]. The $\mathrm{pH}$ of the oral fluid is decreased, that contributes to the accumulation of plaque.

The survey shows that the questions of the questionnaire are understandable for respondents and provide basic subjective information for compiling individual preventive programs [18]. The data collected in the survey show significant differences in the estimated parameters in the groups of patients, which confirm the need to create individual programs to improve effectiveness of the dental disease prevention. Therefore, development of a universal form of an outpatient questionnaire for patients with NAFLD seems extremely relevant. The developed questionnaire is an important tool for quality improvement, since it is intended for use in real work in an outpatient dental reception.

\section{CONCLUSIONS}

So, owing to scientific researches, consideration of the etiopathogenesis of periodontal tissue diseases separately from somatic pathology is impossible. The pathogenesis of periodontal disease in patients with NAFLD is complex and multifactorial. Development and implementation of the questionnaire allow evaluating data on the frequency of occurrence and characteristics of dental complaints, the nature of a diet and oral hygiene in patients with NAFLD.

Future prospectus: The developed methodology has proven its effectiveness and is recommended for implementation in medical institutions in order to reduce the risk factors for dental morbidity in the specified category of patients.

\section{REFERENCES}

1. Jepsen S., Caton J.G., Albandar J.M. et al. Periodontal manifestations of systemic diseases and developmental and acquired conditions: Consensus report of workgroup 3 of the 2017 World Workshop on the Classification of Periodontal and Peri-Implant Diseases and Conditions.J Clin Periodontol. 2018; 45(20):219-S229. doi.org/10.1111/jcpe.12951. 
2. Atezhanov D., Supiev T., Bakiev B. Relationship of Somatic Pathology and Dental Diseases in Children, Prevention Measures and Treatment. Bulletin of Science and Practice. 2019;(8):56-65. doi.org/10.33619/24142948/45/06. (in Russian).

3. Singh S., Gupta K., Garg K.N. et al. Dental Management of the Cardiovascular Compromised Patient: A clinical approach. J Young Pharm. 2017; 9(4):453-456.

4. Pandyarajan V., Gish R.G., Alkhouri N., Noureddin M. Screening for Nonalcoholic Fatty Liver Disease in the Primary Care Clinic. Gastroenterol Hepatol (N Y). 2019;15(7):357-365.

5. Spengler E.K., Loomba R. Recommendations for diagnosis, referral for liver biopsy, and treatment of nonalcoholic fatty liver disease and nonalcoholic steatohepatitis. Mayo Clinic Proceedings. 2015; 90(9):1233-1246.

6. Azzam H., Malnick S. Non-alcoholic fatty liver disease - the heart of the matter. World Journal of Hepatology. 2015; 7(10):1369-1376.

7. Ma X., Liu S., Zhang J. et al. Proportion of NAFLD patients with normal ALT value in overall NAFLD patients:a systematic review and metaanalysis. BMC Gastroenterol. 2020. http://doi.org/10.1186/s12876020-1165-z.

8. Younossi Z., Anstee Q.M., Marietti M. et al. Global burden of NAFLD and NASH: trends, predictions, risk factors and prevention. Nat Rev Gastroenterol Hepatol. 2018; 15:11-20.

9. Sheng X., Che H., Ji Q. et al. The Relationship Between Liver Enzymes and Insulin Resistance in Type 2 Diabetes Patients with Nonalcoholic Fatty Liver Disease. Horm Metab Res. 2018; 50:397-402.

10. Alazawi W., Bernabe E., Tai D. et al. Periodontitis is associated with significant fibrosis in patients with Nonalcoholic Fatty Liver Disease PLoS One. 2017; 12. doi: 10.1371/journal.pone.0185902, PMC5722374.

11. Akinkugbe A., Slade G., Barritt A.S. et al. Periodontitis and Non-alcoholic Fatty Liver Disease, a population based cohort investigation in the Study of Health in Pomerania. J Clin Periodontol. 2017; 44(11):10771087. doi: $10.1111 /$ jcpe. 12800 .

12. Nakahara T., Hyogo H., Ono A. et al. Involvement of Porphyromonas gingivalis in the progression of non-alcoholic fatty liver disease. J Gastroenterol. 2018; 53: 269-280.

13. Yoneda M., Naka S., Nakano K. et al. Involvement of a periodontal pathogen, Porphyromonas gingivalis on the pathogenesis of non-alcoholic fatty liver disease. BMC Gastroenterol. 2012; 12: 16. doi: 10.1186/1471-230X-12-16.

14. Emelyanova N. Relapses of desquamative glossitis in patients with gastroesophageal reflux disease. Biomedical Research and Therapy. 2020; 7(10):4041-4044. doi.org/10.15419/bmrat.v7i10.638.

15. Golla K., Epstein J.B., Cabay R.J. Liver disease: current perspectives on medical and dental management. Oral Surg Oral Med Oral Pathol Oral Radiol Endod. 2004; 98:516-521. doi.org/10.1016/ s1079210404006432.
16. Fadieienko G., Nikiforova Y. Eating disorders and visceral adipose tissue - two interconnected informative markers of prognosis of disorders of nutritional status and risk of development of comorbidity chronic non-communicable diseases. Georgian Med News. 2017; 262:58-64.

17. Emelyanova N., Komir I. Effect of drug-induced pathomorphosis on oral cavity organs and tissues in chronic obstructive pulmonary disease combined with coronary heart disease: A clinical case. Biomedical Research and Therapy. 2021;8(1):4197-4202. doi.org/10.15419/bmrat. v8i1.657.

18. Isayeva G., Rieznik L., Buriakovska 0. et al. The impact of group and individual training on hemodynamics, lipid metabolism, physical activity and quality of life in patients with high and very high cardiovascular risk. Wiad Lek. 2019;72(12):2315-2323.

The article presents the results of the research work of the Division for the Study of the Digestive diseases and its Comorbidity with Noncommunicable Diseases of the Government Institution 'L. T. Malaya Therapy National Institute of the National Academy of Medical Sciences of Ukraine' (Kharkiv, Ukraine).

\section{ORCID and contributionship:}

Dmitry V. Emelyanov: 0000-0002-5597-0456 A,B,C,D,E,F

\section{Conflict of interest:}

The Author declare no conflict of interest.

\section{CORRESPONDING AUTHOR Dmitry V. Emelyanov \\ V. N. Karazin Kharkiv National University 4 Svobody Sq., 61022 Kharkiv, Ukraine tel: +38097-8342429 e-mail:dimadoctorzub@gmail.com}

Received: 24.04 .2020

Accepted: 26.11 .2020

A - Work concept and design, B - Data collection and analysis, C - Responsibility for statistical analysis, 